\title{
Lifetime Maximization of Sensor Networks Under Connectivity and $k$-Coverage Constraints $^{\star}$
}

\author{
Wei Mo, Daji Qiao, and Zhengdao Wang \\ Iowa State University, Ames, IA 50011, USA \\ \{mowei, daji, zhengdao\}@iastate.edu
}

\begin{abstract}
In this paper, we study the fundamental limits of a wireless sensor network's lifetime under connectivity and $k$-coverage constraints. We consider a wireless sensor network with $n$ sensors deployed independently and uniformly in a square field of unit area. Each sensor is active with probability $p$, independently from others, and each active sensor can sense a disc area with radius $r_{s}$. Moreover, considering the inherent irregularity of a sensor's sensing range caused by time-varying environments, we model the sensing radius $r_{s}$ as a random variable with mean $r_{0}$ and variance $r_{0}^{2} \sigma_{s}^{2}$. Two active sensors can communicate with each other if and only if the distance between them is smaller than or equal to the communication radius $r_{c}$.

The key contributions of this paper are: (1) we introduce a new definition of a wireless sensor network's lifetime from a novel probabilistic perspective, called $\omega$-lifetime $(0<\omega<1)$. It is defined as the expectation of the time interval during which the probability of guaranteeing connectivity and $k$-coverage simultaneously is at least $\omega$; and (2) based on the analysis results, we propose a near-optimal scheduling algorithm, called PIS (Pre-planned Independent Sleeping), to achieve the network's maximum $\omega$-lifetime, which is validated by simulation results, and present a possible implementation of the PIS scheme in the distributed manner.
\end{abstract}

\section{Introduction}

Energy conservation is perhaps the most important issue in wireless sensor networks [1, 2]. Most sensor devices are battery-powered and hence have a very limited amount of energy. It is, therefore, very important to extend the battery operation time of individual sensors and, consequently, the network's lifetime. Operating each sensor device in a low duty-cycle has been recognized as an effective way to achieve this goal, where duty-cycle is defined as the fraction of

\footnotetext{
* The research reported in this paper was supported in part by the Information Infrastructure Institute (I-Cube) of Iowa State University. The authors would also like to acknowledge the support from the National Science Foundation under Grants No. ECS 0428040, CCF 0431092, and CNS 0520102. Preliminary results of this research were presented at the 43-rd Annual Allerton Conference on Communication, Control, and Computing, Monticello, IL, Sept. 2005.
} 
time that a sensor device is active. On the other hand, a wireless sensor network typically has two major tasks: sensing and communication. It is always desirable to have all active sensors connected and, at the same time, to have the entire sensing field $k$-covered. The connectivity among active sensors is required in order for an active sensor to report its sensing results back to the user, and the reason for requiring $k$-coverage rather than just 1-coverage is to increase the detection probability and accuracy of tracking. Obviously, the lower the duty-cycle of individual sensors, the longer the wireless sensor network's lifetime, but at the same time, there are a smaller number of active sensors at a given time and, hence, more likely either active sensors are not connected or the $k$-coverage of the sensing field cannot be guaranteed. So, there are inherent tradeoffs, and the key contribution of this paper is to present an integrated study on connectivity, $k$-coverage, and lifetime of a large-scale wireless sensor network.

\subsection{Related Work}

Several researchers $3,4,5,6,7,8$, have addressed the coverage and connectivity issues in wireless sensor/ad hoc networks. Gupta et al. [3] studied scaling laws for asymptotic connectivity of sensors placed at random over a unit area, and provided bounds on connectivity probability for finite-size networks. In 4, the authors presented an analytical procedure to compute the node isolation probability in an ad hoc network in the presence of channel randomness, and showed that, under the assumption that sensing relies on the same wave propagation laws that also guide signal propagation, the coverage probability coincides with the complement of the node isolation probability. In [5], the authors studied the relation between $k$-coverage and $k$-connectivity when the communication radius is at least twice the sensing radius, where the sensing radius is deterministic. However, no statistical properties of either $k$-coverage or $k$-connectivity were given. In [6], three fundamental coverage measures of large-scale sensor networks were studied: area coverage, node coverage, and detectability. In 7 and [8, the asymptotic coverage problem was addressed for mostly-sleeping (unreliable) wireless sensor networks, where 1-coverage was studied in 7] and $k$-coverage in [8, but neither one provided the sufficient-and-necessary condition for asymptotic coverage. None of the above work studied network's lifetime under connectivity and coverage constraints.

Recently, research efforts [9, 10] have been made to analyze the lifetime of a wireless sensor network with coverage requirements. The definitions of network's lifetime in these literature are different from ours. In 9, the lifetime refers to the time it takes for the coverage - defined as the fraction of the area covered by working sensors - to drop below a pre-defined threshold. In [10, the $\alpha$ lifetime of a wireless sensor network is defined as the interval during which at least $\alpha$ portion of the sensing region is covered by at least one sensor node. These lifetime definitions are all from the deterministic point of view, while in this paper, considering the fact that the deployment and dynamics of wireless sensor networks are random and, hence, the coverage of the sensing field and the connectivity among active sensors are also random variables, we study network's 
lifetime from a (different) probabilistic perspective. Moreover, neither [9] nor [10] studied the effect of the communication radius on the network's lifetime.

\subsection{Key Contributions}

This paper explores the fundamental limits of a wireless sensor network's lifetime under connectivity and $k$-coverage constraints, and the contributions are twofold. First, we introduce a new definition of network's lifetime from a probabilistic perspective, namely $\omega$-lifetime, which is defined as the expectation of the time interval during which the probability of guaranteeing connectivity and $k$-coverage simultaneously is at least $\omega$. By solving two convex optimization problems, we obtain a lower bound and an upper bound on the network's maximum $\omega$-lifetime. Second, based on the obtained lower bound, we propose a near-optimal scheduling scheme, called PIS (Pre-planned Independent Sleeping), to maximize the network's $\omega$-lifetime, and describe a possible distributed implementation of the PIS scheme.

\subsection{Organization}

The rest of this paper is organized as follows. Section 2 describes our network model and gives the problem statement. In Section 3, we derive the sufficientand-necessary condition for maintaining $k$-coverage with probability one as the number of sensors goes to infinity. Section 4 describes the details of the proposed $\omega$-lifetime and PIS scheduling scheme. Section 5 presents and evaluates the simulation results and, finally, the paper concludes in Section $\square$.

\section{Network Model and Problem Statement}

\subsection{Network Model}

Consider a wireless sensor network with $n$ sensors deployed independently and uniformly in a square sensing field $\mathcal{D}$ of unit area. In order to extend network's lifetime, an appropriate duty cycle and a well-designed sleeping schedule are required, and we propose the following Pre-planned Independent Sleeping (PIS) scheme for this purpose: time is divided into rounds, and at the beginning of a round, each alive sensor becomes active with probability $p$ or inactive (sleeping) with probability (1-p), independently from others; the value of p and active sensors' communication radius may vary with the round, and their variation patterns are pre-determined by the performance metric to be optimized. Here, alive sensors refer to the sensors with enough energy to operate. The PIS scheme is based on the Randomized Independent Sleeping (RIS) scheme proposed in [8] and the details of PIS will be discussed in Section[4. Note that, in general, RIS-like schemes are energy-efficient, lightweight, and easy to implement because each sensor determines its own sleeping schedule independently without interacting with others. In comparison, the Neighborhood Cooperative Sleeping (NCS) schemes [1, 9, 12,13, allow neighbor sensors to collaborate with each other to determine their sleeping schedules, hence improving the coverage performance further but with increased complexity. Design and analysis of NCS schemes are out of the scope of this paper. 
Sensing model. To consider the sensing radii irregularity caused by timevarying environments, we assume a random sensing radius model where (1) each active sensor has a sensing radius of $r_{s}$; (2) any object within a disc of radius $r_{s}$ centered at an active sensor can be reliably-detected by the sensor; and (3) $r_{s}$ 's are independently identically distributed (i.i.d) random variables with mean $r_{0}$ and variance $r_{0}^{2} \sigma_{s}^{2}$, and the underlying distribution is assumed unknown. A point in the sensing field $\mathcal{D}$ is said to be $k$-covered if it is within the sensing radius of at least $k$ active sensors. The field $\mathcal{D}$ is said to be $k$-covered if every point in $\mathcal{D}$ is $k$-covered.

Communication model. Two active sensors can communicate directly with each other if and only if the distance between them is less than $r_{c}$. The radius $r_{c}$ is referred to as the communication radius and may vary from round to round in the PIS scheme. For the purpose of simplicity, we assume that, at each round, all active sensors have the same and deterministic communication radii. The network is said to be connected if the underlying graph of active sensors is connected. Moreover, we assume torus convention (also known as the toroidal model) 14, i.e., each disc (communication or sensing) that protrudes one side of the field $\mathcal{D}$ enters $\mathcal{D}$ again from the opposite side. This eliminates the edge effects and simplifies the problem.

$\boldsymbol{\omega}$-lifetime. Due to the randomness in sensor deployment and sleeping schedule, it is impossible to guarantee connectivity and $k$-coverage with probability one with finite number of sensors, unless the communication disc and the sensing disc of each active sensor can cover the entire field. However, the physical limitations prohibit such large communication radius and sensing radius. In other words, there is no deterministic guarantee of connectivity or $k$-coverage for randomly-deployed wireless sensor networks in practice. Such facts motivate us to study the network's lifetime from a probabilistic perspective. More specifically, we define the $\omega$-lifetime of a randomly-deployed wireless sensor network as the expectation of the time interval during which the probability of guaranteeing $k$-coverage of field $\mathcal{D}$ and the connectivity of the network simultaneously is at least $\omega$, where $0<\omega<1$. For example, suppose that the PIS scheduling scheme is employed, then the network's $\omega$-lifetime is $T_{\omega}=\mathrm{E}\left[\sum_{i=1}^{M} T_{i}\right]$, where $T_{i}$ is the duration of the $i$-th round, and $M$ is the maximum number of rounds during which the network can function properly. In other words, for any round $i$ $(i \leqslant M)$, the probability of guaranteeing both connectivity and $k$-coverage simultaneously, denoted by $P_{\mathrm{c} \& \mathrm{c}}$, is at least $\omega$, but for round $(M+1), P_{\mathrm{c} \& \mathrm{c}}$ is smaller than $\omega$.

\subsection{Problem Statement}

The kernel problem we study in this paper is:

- For a finite-size wireless sensor network, how to find the optimal parameters $\left(p\right.$ and $\left.r_{c}\right)$ for the PIS scheme to maximize the $\omega$-lifetime of the network? 
This is an interesting problem and the results may serve as good guidelines in deploying finite-size wireless sensor networks. In order to address this problem, we first study the following companion problem, which is referred to as the critical condition for asymptotic $k$-coverage:

- What relation among $n, p, r_{0}$, and $\sigma_{s}^{2}$ would be the sufficient-and-necessary condition to guarantee that the probability of the entire field $\mathcal{D}$ being $k$ covered approaches 1 as $n$ goes to infinity?

\section{Critical Condition for Asymptotic $k$-Coverage}

In this section, we derive the sufficient-and-necessary condition, under our random sensing radius model described in Section 2.1 to guarantee that the entire sensing field $\mathcal{D}$ is $k$-covered with probability one as the total number of deployed sensors $n$ goes to infinity. Similar to [10, we apply the coverage process techniques introduced in [14] to solve the problem.

Lemma 1. Let $n$ points distributed independently and uniformly in a square field $\mathcal{D}$ of unit area within $\mathbb{R}^{2}$, then for sufficiently large $n$, these points form a stationary Poisson point process with density $n$.

Lemma 1 is a well-known result and its proof is given by Hall in 14. Let $\mathcal{P} \equiv$ $\left\{\xi_{i}, i \geqslant 1\right\}$ denote the set of active sensors. It is shown in Lemma 2 that $\mathcal{P}$ is also a stationary Poisson point process with density $n p$ for sufficiently large $n$.

Lemma 2. Let $n$ points distributed independently and uniformly in a square field $\mathcal{D}$ of unit area within $\mathbb{R}^{2}$. Each point is marked independently as an active point with probability $p$, where $0<p \leqslant 1$. Then the set of active points, $\mathcal{P}=$ $\left\{\xi_{i}, i \geqslant 1\right\}$, is a stationary Poisson point process with density np for sufficiently large $n$.

Let $S_{i}$ denote a random disc with radius $r_{s, i}$ centered at the origin of $\mathbb{R}^{2}$, which is defined as $S_{i} \equiv\left\{x \in \mathbb{R}^{2}:|x| \leqslant r_{s, i}\right\}$, where $r_{s, i}$ is the sensing radius of the $i$-th active sensor $\xi_{i}$. Here, we assume that all sensing radii are i.i.d random variables following an unknown distribution $F(r)$, with known mean $r_{0}$ and variance $r_{0}^{2} \sigma_{s}^{2}$, i.e., all $S_{i}$ 's are distributed as $S$ :

$$
S \equiv\left\{x \in \mathbb{R}^{2}:|x| \leqslant r, r \sim F(r)\right\} .
$$

Then, the sensing disc (abbreviated as disc) centered at active sensor $\xi_{i}$ can be defined as $D_{i} \equiv \xi_{i}+S_{i}=\left\{\xi_{i}+y: y \in S_{i}\right\}$. The set of $\left\{D_{i}, i \geqslant 1\right\}$ forms a stationary coverage process. For such a coverage process, Lemma 3 gives the distribution of the number of discs with certain properties.

Lemma 3. Let $\mathcal{Q}=\left\{\xi_{i}+S_{i}, i \geqslant 1\right\}$ denote a stationary coverage process, where $\left\{\xi_{i}\right\}$ is a stationary Poisson point process with density $\lambda$ within $\mathcal{D}$, and $S_{i}$ 's are distributed as $S$ defined in (11). For a given deterministic condition $C$, let 
$Y$ denote the number of discs in $\mathcal{Q}$ that satisfy the condition $C$. Then, $Y$ is Poisson-distributed with mean

$$
\mu=\lambda \cdot \mathrm{E}\left[\left\|\left\{x: I_{C}(x+S)=1\right\}\right\|\right],
$$

where $I_{C}(\cdot)$ is the indicator function of whether a disc satisfies the condition $C$ or not, and $\|\cdot\|$ denotes the area.

The proofs of Lemma 2 and Lemma 3 are omitted due to space limitation. Interested readers can refer to the full version of this paper [15].

Let $Y(x)$ denote the number of active sensors that cover a point $x$, and $I_{k}(x)$ denote the indicator function of whether the point $x$ is covered by at most $(k-1)$ active sensors, i.e.,

$$
I_{k}(x)= \begin{cases}1, & \text { if } Y(x)<k \\ 0, & \text { otherwise }\end{cases}
$$

Then, the expectation of Bernoulli random variable $I_{k}(x)$ is

$$
\mathrm{E}\left[I_{k}(x)\right]=P(x \text { is at most }(k-1) \text {-covered })=P(Y(x)<k) .
$$

By Lemma 3, we know that $Y(x)$ is Poisson-distributed with mean

$$
\mu=n p \cdot \mathrm{E}[\|\{x:(x+S) \cap\{x\} \neq \emptyset\}\|]=n p \cdot \mathrm{E}[\|x-S\|]=n p a_{s},
$$

where $a_{s} \equiv \mathrm{E}[\|S\|]=\pi r_{0}^{2}\left(1+\sigma_{s}^{2}\right)$. Therefore,

$$
\mathrm{E}\left[I_{k}(x)\right]=e^{-n p a_{s}} \sum_{j=0}^{k-1} \frac{\left(n p a_{s}\right)^{j}}{j !} .
$$

Let the $k$-vacancy $V_{k}$ denote the area within $\mathcal{D}$ that is covered by at most $(k-1)$ active sensors, then the random variable $V_{k}$ can be expressed as $V_{k}=$ $\int_{\mathcal{D}} I_{k}(x) \mathrm{d} x$. Using Fubini's theorem [16] and exchanging the order of integral and expectation, we obtain the expected value of the $k$-vacancy as:

$$
\mathrm{E}\left[V_{k}\right]=\int_{\mathcal{D}} \mathrm{E}\left[I_{k}(x)\right] \mathrm{d} x=e^{-n p a_{s}} \sum_{j=0}^{k-1} \frac{\left(n p a_{s}\right)^{j}}{j !} .
$$

$K$-coverage of the sensing field $\mathcal{D}$ means that each point in $\mathcal{D}$ should be covered by at least $k$ active sensors, which implies $V_{k}=0$. Because sensors are deployed independently and uniformly within $\mathcal{D}$, it cannot guarantee $P\left(V_{k}=0\right)=1$ with finite $n$ for $a_{s}<1$ regardless of the value of $n$. However, if $n p \rightarrow \infty$ as $n \rightarrow \infty$, it is possible that $P\left(V_{k}=0\right) \rightarrow 1$ as $n \rightarrow \infty$. Before studying the asymptotic behavior of $P\left(V_{k}=0\right)$, we first give an upper bound and a lower bound on $P\left(V_{k}=0\right)$ for finite $n$. Similar bounds have been proved in [10] for the case of deterministic sensing radius model and non-sleeping sensor networks. Theorem 1 is a generalization of the results in [10] for the random sensing radius model. 
Theorem 1. For $n>1,0<p \leqslant 1$, and $a_{s}<1$,

$$
P_{l}<P\left(V_{k}=0\right)<P_{u}
$$

in which

$$
P_{u}=\frac{4(k+1) !\left(1+\sigma_{s}^{2}\right)(n p)^{-1}\left(n p a_{s}\right)^{-k} \cdot e^{n p a_{s}}}{1+4(k+1) !\left(1+\sigma_{s}^{2}\right)(n p)^{-1}\left(n p a_{s}\right)^{-k} \cdot e^{n p a_{s}}}
$$

and

$$
P_{l}=1-2 e^{-n p a_{s}}\left(1+\left(n^{2} p^{2} a_{s}^{\prime}+2 n p r_{0}\right) \sum_{i=0}^{k-1} \frac{\left(n p a_{s}\right)^{i}}{i !}\right)
$$

where $a_{s}^{\prime} \equiv \pi r_{0}^{2}\left(1+\sigma_{s}^{2} / 2\right)$.

Proof: (i) Upper bound.

By the Cauchy-Schwartz inequality [14,

$$
\mathrm{E}\left[V_{k}\right]=\mathrm{E}\left[V_{k} \cdot I\left(V_{k}>0\right)\right] \leqslant\left\{\mathrm{E}\left[V_{k}^{2}\right] P\left(V_{k}>0\right)\right\}^{1 / 2},
$$

where $I(\cdot)$ denotes the indicator function, thus

$$
P\left(V_{k}>0\right) \geqslant \frac{\left(\mathrm{E}\left[V_{k}\right]\right)^{2}}{\mathrm{E}\left[V_{k}^{2}\right]}
$$

where $\mathrm{E}\left[V_{k}^{2}\right]=\mathrm{E}\left[\iint_{\mathcal{D}^{2}} I_{k}\left(x_{1}\right) I_{k}\left(x_{2}\right) d x_{1} d x_{2}\right]=\iint_{\mathcal{D}^{2}} \mathrm{E}\left[I_{k}\left(x_{1}\right) I_{k}\left(x_{2}\right)\right] d x_{1} d x_{2}$. Let $Y_{1}$ denote the number of active sensors that cover $x_{1}, Y_{2}$ the number of active sensors that cover $x_{2}$, and $Y_{3}$ the number of active sensors that cover $x_{2}$ but not $x_{1}$, then

$$
\mathrm{E}\left[I_{k}\left(x_{1}\right) I_{k}\left(x_{2}\right)\right]=P\left(Y_{1}<k, Y_{2}<k\right) \leqslant P\left(Y_{1}<k, Y_{3}<k\right) .
$$

Lemma 4. For the random variables $Y_{1}$ and $Y_{3}$ defined above, we have the following results:

- $Y_{1}$ is Poisson-distributed with mean npa $_{s}$,

$-Y_{3}$ is Poisson-distributed with mean $n p b_{s}$,

- $Y_{1}$ and $Y_{3}$ are independent,

where $b_{s} \equiv \mathrm{E}\left[\left\|\left\{x:(x+S) \cap\left\{x_{1}\right\}=\emptyset,(x+S) \cap\left\{x_{2}\right\} \neq \emptyset\right\}\right\|\right]$.

The proof of Lemma 4 is omitted here due to space limitation. Interested readers can refer to [15]. Using Lemma 4 and (8), we have

$$
\begin{aligned}
\mathrm{E}\left[I_{k}\left(x_{1}\right) I_{k}\left(x_{2}\right)\right] & \leqslant P\left(Y_{1}<k\right) \cdot P\left(Y_{3}<k\right)=\mathrm{E}\left[I_{k}\left(x_{1}\right)\right] \cdot P\left(Y_{3}<k\right) \\
& =\mathrm{E}\left[I_{k}\left(x_{1}\right)\right] \cdot\left(e^{-n p b_{s}} \sum_{j=0}^{k-1} \frac{\left(n p b_{s}\right)^{j}}{j !}\right) .
\end{aligned}
$$


Let $z=x_{1}-x_{2}$, then

$$
\begin{aligned}
b_{s} & =\mathrm{E}\left[\left\|\left\{x:(x+S) \cap\left\{x_{1}\right\}=\emptyset,(x+S) \cap\left\{x_{2}\right\} \neq \emptyset\right\}\right\|\right] \\
& =\mathrm{E}[\|\{x:(x+S) \cap\{z\}=\emptyset,(x+S) \cap\{0\} \neq \emptyset\}\|]=a_{s}-\rho(z),
\end{aligned}
$$

where

$$
\begin{aligned}
& \rho(z)=\mathrm{E}[\|\{x:(x+S) \cap\{z\} \neq \emptyset,(x+S) \cap\{0\} \neq \emptyset\}\|]=\int_{0}^{\infty} r^{2} B(|z| / 2 r) d F(r), \\
& \text { and }
\end{aligned}
$$

$$
B(x)= \begin{cases}4 \int_{x}^{1} \sqrt{\left(1-y^{2}\right)} d y & \text { if } 0 \leqslant x \leqslant 1, \\ 0 & \text { otherwise }\end{cases}
$$

is the area of the lens of intersection of two unit discs centered $2 x$ apart, and $F(r)$ is the distribution of sensing radius $r_{s}$.

It is shown in 15 that $B(x) \leqslant \pi(1-x)$ for $0 \leqslant x \leqslant 1$, then using the fact that $\rho(z) \geqslant 0$ and after some algebraic manipulation, we can bound $\rho(z)$ as

$$
\begin{cases}\rho(z) \leqslant a_{s}-\pi r_{0}|z| / 2 & \text { if }|z|<2 r_{0}\left(1+\sigma_{s}^{2}\right), \\ \rho(z)=0 & \text { if }|z| \geqslant 2 r_{0}\left(1+\sigma_{s}^{2}\right) .\end{cases}
$$

If $|z| \geqslant 2 r_{0}\left(1+\sigma_{s}^{2}\right)$, then $b_{s}=a_{s}$. Using (9), we have

$$
\mathrm{E}\left[I_{k}\left(x_{1}\right) I_{k}\left(x_{2}\right)\right] \leqslant \mathrm{E}\left[I_{k}\left(x_{1}\right)\right] \cdot \mathrm{E}\left[I_{k}\left(x_{2}\right)\right] .
$$

Therefore,

$$
\begin{aligned}
I_{1} & \equiv \iint_{\mathcal{D}^{2} \cap\left\{\left|x_{1}-x_{2}\right| \geqslant 2 r_{0}\left(1+\sigma_{s}^{2}\right)\right\}} \mathrm{E}\left[I_{k}\left(x_{1}\right) I_{k}\left(x_{2}\right)\right] d x_{1} d x_{2} \\
& \leqslant \iint_{\mathcal{D}^{2}} \mathrm{E}\left[I_{k}\left(x_{1}\right)\right] \cdot \mathrm{E}\left[I\left(x_{2}\right)\right] d x_{1} d x_{2}=\left(\mathrm{E}\left[V_{k}\right]\right)^{2} .
\end{aligned}
$$

Similarly, if $|z|<2 r_{0}\left(1+\sigma_{s}^{2}\right)$, then $b_{s} \geqslant \pi r_{0}|z| / 2$. Using (9), we have

$$
\mathrm{E}\left[I_{k}\left(x_{1}\right) I_{k}\left(x_{2}\right)\right] \leqslant \mathrm{E}\left[I_{k}\left(x_{1}\right)\right] \cdot\left(e^{-n p \frac{\pi r_{0}}{2}|z|} \sum_{j=0}^{k-1} \frac{\left(n p \pi r_{0}|z|\right)^{j}}{2^{j} \cdot j !}\right) .
$$

Therefore,

$$
\begin{aligned}
I_{2} & \equiv \iint_{\mathcal{D}^{2} \cap\left\{\left|x_{1}-x_{2}\right|<2 r_{0}\left(1+\sigma_{s}^{2}\right)\right\}} \mathrm{E}\left[I_{k}\left(x_{1}\right) I_{k}\left(x_{2}\right)\right] d x_{1} d x_{2} \\
& \leqslant \int_{\mathcal{D}} \mathrm{E}\left[I_{k}\left(x_{1}\right)\right] d x_{1} \int_{0}^{2 r_{0}\left(1+\sigma_{s}^{2}\right)} e^{-n p \pi r_{0} z / 2} \sum_{i=0}^{k-1} \frac{\left(n p \pi r_{0} z\right)^{i}}{2^{i} \cdot i !} 2 \pi z d z
\end{aligned}
$$




$$
\begin{aligned}
& =\mathrm{E}\left[V_{k}\right] \cdot\left(\int_{0}^{1} e^{-\lambda u} \sum_{i=0}^{k-1} \frac{(\lambda u)^{i}}{i !} 8 \pi r_{0}^{2}\left(1+\sigma_{s}^{2}\right)^{2} u d u\right) \\
& <4 a_{s}\left(1+\sigma_{s}^{2}\right) k(k+1) \lambda^{-2},
\end{aligned}
$$

where $\lambda=n p a_{s}$. The proof of the last inequality above can be found in [15]. Hence, we have

$$
I_{2}<4 a_{s}\left(1+\sigma_{s}^{2}\right) k(k+1)\left(n p a_{s}\right)^{-2} \cdot\left(e^{-n p a_{s}} \sum_{i=0}^{k-1} \frac{\left(n p a_{s}\right)^{i}}{i !}\right) .
$$

Since $\mathrm{E}\left[V_{k}^{2}\right]=I_{1}+I_{2}$, combining (77), (3), (10), and (11), we can upper-bound $P\left(V_{k}=0\right)$ as follows:

$$
P\left(V_{k}=0\right)=1-P\left(V_{k}>0\right) \leqslant 1-\frac{\left(\mathrm{E}\left[V_{k}\right]\right)^{2}}{\mathrm{E}\left[V_{k}^{2}\right]}<\frac{\beta}{1+\beta},
$$

where

$$
\beta=\frac{4\left(1+\sigma_{s}^{2}\right) a_{s} k(k+1)\left(n p a_{s}\right)^{-2}}{e^{-n p a_{s}} \sum_{i=0}^{k-1}\left(n p a_{s}\right)^{i} / i !} \leqslant 4\left(1+\sigma_{s}^{2}\right)(k+1) !(n p)^{-1}\left(n p a_{s}\right)^{-k} \cdot e^{n p a_{s}} .
$$

Therefore, we obtain the upper bound on $P\left(V_{k}=0\right)$ as

$$
P\left(V_{k}=0\right)<\frac{4(k+1) !\left(1+\sigma_{s}^{2}\right)(n p)^{-1}\left(n p a_{s}\right)^{-k} \cdot e^{n p a_{s}}}{1+4(k+1) !\left(1+\sigma_{s}^{2}\right)(n p)^{-1}\left(n p a_{s}\right)^{-k} \cdot e^{n p a_{s}}} .
$$

(ii) Lower bound.

Observe that

$$
p\left(V_{k}=0\right)=1-p_{1}-p_{2}-p_{3},
$$

where

$$
p_{1}=P(\text { no active sensors centered within } \mathcal{D})=e^{-n p}<e^{-n p a_{s}} .
$$

Here, we assume $a_{s}<1$, meaning that, even for the random sensing radius model, the expected sensing area of one sensor will not cover the entire field $\mathcal{D}$.

$p_{2}=P($ at least one disc centered within $\mathcal{D}$, but none of the discs intersects with any other disc, and none of the discs intersect the boundary of $\mathcal{D}$ )

$\leqslant P($ at least one disc centered within $\mathcal{D}) \cdot P($ a given disc intersects with no other discs $)$

$$
=\left(1-e^{-n p}\right) \cdot e^{-n p \pi \mathrm{E}\left[\pi\left(r_{s, 1}+r_{s, 2}\right)^{2}\right]}=\left(1-e^{-n p}\right) \cdot e^{-2 n p \pi r_{0}^{2}\left(2+\sigma_{s}^{2}\right)}<e^{-n p a_{s}},
$$

where $r_{s, 1}$ and $r_{s, 2}$ are sensing radii of two active sensors, which are i.i.d with mean $r_{0}$ and variance $r_{0}^{2} \cdot \sigma_{s}^{2}$, and the second equality is due to Lemma 3 ,

$p_{3}=P(\mathcal{D}$ is not $k$-covered, at least one disc centered within $\mathcal{D}$, and at least one disc intersects with another disc or the boundary of $\mathcal{D}$ ). 
Therefore

$$
p\left(V_{k}=0\right)>1-2 e^{-n p a_{s}}-p_{3} .
$$

Our next task is to derive an upper bound on $p_{3}$.

Define a crossing to be either an intersection point of the boundaries of two discs or an intersection point of the boundary of an disc and the boundary of the field $\mathcal{D}$. A crossing is said to be $k$-covered if it is within at least $k$ discs. It is proved in [5] that, field $\mathcal{D}$ is $k$-covered if there exist crossings and every crossing is $k$-covered. Therefore, if $\mathcal{D}$ is not $k$-covered, if one or more discs are centered within $\mathcal{D}$, and if there exist crossings in $\mathcal{D}$, then at least one of the discs has two or more crossings that are not $k$-covered. Thus

$$
p_{3} \leqslant P\left(M_{k} \geqslant 2\right) \leqslant \mathrm{E}\left[M_{k}\right] / 2,
$$

where $M_{k}$ denotes the number of crossings that are not $k$-covered.

Define $L_{1}$ and $L_{2}$ as the number of crossings created by two discs intersecting with each other, and the ones created by a disc intersecting the boundary of field $\mathcal{D}$. We first study the expected value of $L_{1}$. The expected number of crossings created by a given active sensor $\xi_{1}$ with other active sensors is

$$
\mathrm{E}\left[2 n p \cdot \pi\left(r_{s, 1}+r_{s, 2}\right)^{2}\right]=8 n p a_{s}^{\prime},
$$

where $a_{s}^{\prime} \equiv \pi r_{0}^{2}\left(1+\sigma_{s}^{2} / 2\right)$, and the expected number of discs centered within $\mathcal{D}$ is $n p$. Therefore,

$$
\mathrm{E}\left[L_{1}\right]=n p \cdot 8 n p a_{s}^{\prime} / 2=4 n^{2} p^{2} a_{s}^{\prime} .
$$

If a disc intersects the edge of field $\mathcal{D}$, at most two crossings will be created; if a disc intersects the corner of field $\mathcal{D}$, at most four crossings will be created (due to the toroidal model assumption). Thus the expected value of $L_{2}$ is bounded by

$$
\mathrm{E}\left[L_{2}\right] \leqslant 8 n p r_{0} .
$$

The probability that a given crossing is not $k$-covered is given by (2) . Therefore,

$$
\mathrm{E}\left[M_{k}\right]=\left(\mathrm{E}\left[L_{1}\right]+\mathrm{E}\left[L_{2}\right]\right) e^{-n p a_{s}} \sum_{j=0}^{k-1} \frac{\left(n p a_{s}\right)^{j}}{j !} \leqslant 4\left(n^{2} p^{2} a_{s}^{\prime}+2 n p r_{0}\right) e^{-n p a_{s}} \sum_{j=0}^{k-1} \frac{\left(n p a_{s}\right)^{j}}{j !} .
$$

By (12), (13), and (14), we have

$$
P\left(V_{k}=0\right)>1-2 e^{-n p a_{s}}\left(1+\left(n^{2} p^{2} a_{s}^{\prime}+2 n p r_{0}\right) \sum_{i=0}^{k-1} \frac{\left(n p a_{s}\right)^{i}}{i !}\right) .
$$

This completes the proof.

In what follows, we establish the sufficient-and-necessary condition for asymptotic $k$-coverage. 
Corollary 1. Assume $n p \rightarrow \infty$ as $n \rightarrow \infty$, and let

$$
\pi r_{0}^{2}\left(1+\sigma_{s}^{2}\right)=\frac{\ln (n p)+k \ln \ln (n p)+c_{1}(n p)}{n p},
$$

then the entire unit square field $\mathcal{D}$ is $k$-covered with probability one as $n \rightarrow \infty$, if and only if $c_{1}(n p) \rightarrow \infty$ as $n \rightarrow \infty$.

Proof: The entire unit square field $\mathcal{D}$ is $k$-covered with probability one means that $P\left(V_{k}=0\right) \rightarrow 1$ as $n \rightarrow \infty$. First, we prove if $c_{1}(n p) \rightarrow \infty$ as $n \rightarrow \infty$, $P\left(V_{k}=0\right) \rightarrow 1$.

By (4) and (6) in Theorem 11, we have

$$
P\left(V_{k}=0\right)>1-2 e^{-n p a_{s}}-\left(b_{1}+b_{2}\right) \cdot(n p)\left(n p a_{s}\right)^{k} e^{-n p a_{s}},
$$

where $b_{1} \equiv 2 k \frac{1+\sigma_{s}^{2} / 2}{1+\sigma_{s}^{2}}>0$ is independent of $n$, and $b_{2} \equiv \frac{4 k}{\pi r_{0}\left(1+\sigma_{s}^{2}\right) n p}$. Let $n p a_{s}=$ $\ln (n p)+k \ln \ln (n p)+c_{1}(n p)$, then $n p a_{s} \rightarrow \infty, e^{-n p a_{s}} \rightarrow 0$, and $b_{2} \rightarrow 0$, as $n \rightarrow \infty$. Therefore, when $c_{1}(n p) \rightarrow \infty$,

$$
\begin{aligned}
\ln \left(\left(b_{1}+b_{2}\right) \cdot(n p)\left(n p a_{s}\right)^{k} e^{-n p a_{s}}\right)= & \ln \left(b_{1}+b_{2}\right)-k \ln \ln (n p)-c_{1}(n p) \\
& +k \cdot \ln \left(\ln (n p)+k \ln \ln (n p)+c_{1}(n p)\right) \\
\rightarrow & -\infty,
\end{aligned}
$$

and consequently, $P\left(V_{k}=0\right) \rightarrow 1$. The first part is proved.

If $c_{1}(n p) \leqslant C_{1}$ for some finite $C_{1}>0$ as $n \rightarrow \infty$, then for sufficiently large $n$,

$$
\begin{aligned}
4(k+1) !\left(1+\sigma_{s}^{2}\right)(n p)^{-1}\left(n p a_{s}\right)^{-k} e^{n p a_{s}} & =4(k+1) !\left(1+\sigma_{s}^{2}\right) e^{c_{1}(n p)} \\
& \leqslant 4 e^{C_{1}}(k+1) !\left(1+\sigma_{s}^{2}\right) .
\end{aligned}
$$

Therefore, by (4) and (5), we have

$$
P\left(V_{k}=0\right)<\frac{4 e^{C_{1}}(k+1) !\left(1+\sigma_{s}^{2}\right)}{1+4 e^{C_{1}}(k+1) !\left(1+\sigma_{s}^{2}\right)}<1 .
$$

It means that $P\left(V_{k}=0\right) \rightarrow 1$ only if $c_{1}(n p) \rightarrow \infty$ as $n \rightarrow \infty$. This completes the proof.

Remark: The bounds obtained in Theorem 1 is valid for finite $n$. Therefore, they can be used as performance criteria for designing finite-size sensor networks, as will be shown in the next section.

\section{$4 \omega$-lifetime of Finite-Size Wireless Sensor Networks}

In this section, we address the problem of finding optimal parameters for the PIS scheme to maximize the $\omega$-lifetime of a finite-size wireless sensor network.

Let $A$ denote the event of the sensing field $\mathcal{D}$ being $k$-covered, and $B$ denote the event of the sensor network being connected. The probability of guaranteeing simultaneously $k$-coverage of field $\mathcal{D}$ and connectivity of the network is $P_{\mathrm{c} \& \mathrm{c}} \equiv$ $P(A \cap B)$. 
Definition 1. $\omega$-lifetime, denoted by $T_{\omega}$, of a sensor network is defined as the expectation of the time interval during which the probability of guaranteeing simultaneously $k$-coverage of field $\mathcal{D}$ and the connectivity of the network is no less than $\omega$, i.e., $P_{\mathrm{c} \& \mathrm{c}} \geqslant \omega$, where $0<\omega<1$.

In order to study the $\omega$-lifetime, we first introduce the energy consumption model of each wireless sensor. We assume that inactive sensors do not consume energy and the communication traffic is evenly distributed across the network. The energy consumption of an active sensor consists of two parts: communication and sensing. Thus, the power consumption $P_{0}$ of each active sensor can be modeled as

$$
P_{0}=Q \cdot \frac{1}{r_{c}} \cdot r_{c}^{\beta}+\Delta,
$$

where

$-r_{c}^{\beta}$ is proportional to the communication energy consumption per bit, and the typical values of $\beta$ range from 3 to 4 for different propagation models [17;

$-1 / r_{c}$ is proportional to the average traffic rate of active sensors (we assume that all active sensors have the same traffic rate, following the assumption of evenly distributed traffic.);

$-\Delta$ is the power consumption for continuous sensing and listening;

$-Q>0$ is a constant.

As the communication radius $r_{c}$ decreases, the average number of hops required for packets transmitted from one point to another increases inversely. For this reason, we incorporate the factor of $1 / r_{c}$ into the average traffic rate expression. We further assume that all active sensors have the same communication radius $r_{c}$, which results in the same individual lifetime:

$$
T_{0}\left(r_{c}\right)=\frac{E_{0}^{\prime}}{P_{0}}=\frac{E_{0}}{r_{c}^{\beta-1}+\eta}
$$

where $E_{0}^{\prime}$ is the initial energy of each active sensor, $E_{0}=\frac{E_{0}^{\prime}}{Q}$, and $\eta=\frac{\Delta}{Q}$, respectively. This assumption is typical when analyzing the network's lifetime, e.g., in [10] and [18].

Next, we formally define the PIS scheme which can extend the $\omega$-lifetime of a wireless sensor network. Suppose that time is divided into rounds. At the beginning of round $i$, there are $n^{(i)}$ alive sensors, and each alive sensor decides independently whether to remain sleeping (with probability $1-p^{(i)}$ ), or become active (with probability $p^{(i)}$ ). All active sensors choose the same communication radius of $r_{c}^{(i)}$. Both $p^{(i)}$ and $r_{c}^{(i)}$ are chosen such that $P_{c \& c} \geqslant \omega$. Next, all active sensors will operate continuously until batteries die out. Since we assume that all active sensors have the same individual lifetime, they will die out at the same time instant, which is defined as the end of this round. The same procedure is repeated for the next rounds until there are not enough alive sensors to satisfy the " $P_{\mathrm{c} \& c} \geqslant \omega$ " requirement, regardless of the choices of $p$ and $r_{c}$. 
The major differences between PIS and RIS in [8] are as follows. In PIS, $p$ and $r_{c}$ are chosen for each round to satisfy both connectivity and $k$-coverage requirements, and they may vary from round to round. The round duration is the same as an individual sensor's lifetime, i.e., within each round, all active sensors operate continuously until batteries die out. In comparison, the round duration of the RIS scheme is selected to be sufficiently-small, and the values of $p$ and $r_{c}$ in RIS are fixed throughout the network operation, where $p$ is chosen to satisfy the $k$-coverage requirement but with no optimization on $r_{c}$. This way, batteries of all sensors die out at approximately the same time around the end of the network's lifetime.

In the rest of this section, we study the $\omega$-lifetime with the proposed PIS scheme and try to find the optimal parameters to maximize the $\omega$-lifetime of the network.

\section{$4.1 \omega$-Lifetime Study}

Suppose that $n$ sensors are deployed independently and uniformly within a unitarea square field $\mathcal{D}$, and the network can operate $M$ rounds following the PIS scheduling scheme. Then, the $\omega$-lifetime of the wireless sensor network is

$$
T_{\omega}=\mathrm{E}\left[\sum_{i=1}^{M} T_{0}\left(r_{c}^{(i)}\right)\right]=\mathrm{E}\left[\sum_{i=1}^{M} \frac{E_{0}}{\left(r_{c}^{(i)}\right)^{\beta-1}+\eta}\right],
$$

subject to both connectivity and $k$-coverage requirements, and the expectation is with respect to $M$. Define $n_{\text {eff }}^{(i)}=n^{(i)} p^{(i)}$, which is the expected number of active sensors in round $i$. It is easy to verify that the probability mass function (pmf) of $M$ is

$$
P(M=m)=\sum_{\substack{n=n^{(1)} \geqslant n^{(2)} \geqslant \ldots \geqslant n(m) \\
n^{(i)} \geqslant n_{\text {eff }}^{(i)}, i=1, \ldots, m}} \cdots \sum_{n^{(m+1)}=0} \prod_{\substack{n_{\text {eff }}^{(m+1)}-1 \\
m}}\left(\begin{array}{c}
n^{(i)} \\
n^{(i+1)}
\end{array}\right)\left(1-p^{(i)}\right)^{n^{(i+1)}}\left(p^{(i)}\right)^{n^{(i)}-n^{(i+1)}} .
$$

Thus, the problem of maximizing the $\omega$-lifetime of the network can be expressed as

$$
\begin{aligned}
& T_{\omega}^{\max }=\max _{r_{c}^{(i)}, n_{\mathrm{eff}}^{(i)}} T_{\omega}=\max _{r_{c}^{(i)}, n_{\mathrm{eff}}^{(i)}} \mathrm{E}\left[\sum_{i=1}^{M} \frac{E_{0}}{\left(r_{c}^{(i)}\right)^{\beta-1}+\eta}\right], \\
& \text { subject to } P_{\mathrm{c} \& \mathrm{c}}=P(A \cap B) \geqslant \omega \text { for each round. }
\end{aligned}
$$

Using the union bound, we have

$$
\min \{P(A), P(B)\} \geqslant P_{\mathrm{c} \& \mathrm{c}} \geqslant P(A)+P(B)-1 .
$$

Since it is hard to analyze $P_{c \& c}$ directly, we next focus on finding a lower bound and an upper bound on the optimal $\omega$-lifetime, $T_{\omega}^{\max }$. 
Lower bound. Restricting the constraint in (20) by replacing it with the lower bound in (21), and assuming that all $n_{\text {eff }}^{(i)}$ and $r_{c}^{(i)}$, s are the same for each round, we can obtain a lower bound on $T_{\omega}^{\max }$ by solving the following optimization problem:

$$
\begin{gathered}
\max _{n_{\mathrm{eff}}, r_{c}, \epsilon} \mathrm{E}[M] \cdot \frac{E_{0}}{r_{c}^{\beta-1}+\eta}, \\
\text { subject to } P(A) \geqslant \omega+\epsilon, P(B) \geqslant 1-\epsilon \text { for } 0<\epsilon<1-\omega .
\end{gathered}
$$

Using the result $P(A)>P_{l}$ in Theorem 1, and the following result in [3]:

$$
P(B) \approx 1-P(\exists \text { isolated active sensors })>1-n_{\mathrm{eff}} e^{-n_{\mathrm{eff}} \pi r_{c}^{2}},
$$

where the edge effects are avoided by the toroidal model assumption, we can restrict the constraints in (23) as

$$
P_{l} \geqslant \omega+\epsilon, r_{c} \geqslant \sqrt{\left[\ln \left(n_{\mathrm{eff}} / \epsilon\right)\right] /\left(\pi n_{\mathrm{eff}}\right)} \text { for } 0<\epsilon<1-\omega
$$

Notice that the value of $\omega$ is usually larger than $90 \%$ in practice, then the $P_{l}$ defined in (6) can be approximated as

$$
P_{l} \approx 1-g\left(n_{\mathrm{eff}}\right) \equiv 1-2 n_{\mathrm{eff}}^{2} a_{s}^{\prime} e^{-a_{s} n_{\mathrm{eff}}} \sum_{i=0}^{k-1} \frac{\left(a_{s} n_{\mathrm{eff}}\right)^{i}}{i !}
$$

Let $X_{i}$ denote the number of active sensors in round $i$, then $n^{(m)}=n-$ $\sum_{i=1}^{m-1} X_{i}$, and conditional on $n^{(i)}, X_{i}$ is $\operatorname{Binomial}$-distributed as $\operatorname{BIN}\left(n^{(i)}, p^{(i)}\right)$. Next, we use the expectation of $n^{(i)}$ to obtain an approximation of $p^{(i)}$ as

$$
p^{(i)}=\frac{n_{\mathrm{eff}}}{n^{(i)}} \approx \frac{n_{\mathrm{eff}}}{n-(i-1) n_{\mathrm{eff}}}=\frac{1}{M_{0}+1-i},
$$

where $M_{0} \equiv n / n_{\text {eff. Using (26) }}$ and the central limit theorem, we can approximate $n^{(m)}$ as a Gaussian random variable with mean $n-(m-1) n_{\mathrm{eff}}$ and variance $A(m) n_{\text {eff }}$, where $A(m)=\sum_{i=1}^{m-1}\left(1-p^{(i)}\right)$. Then, we have

$$
\begin{aligned}
& P(M \leqslant m)=P\left(n^{(m+1)}<n_{\mathrm{eff}}\right)=Q\left(\frac{n-(m+1) n_{\mathrm{eff}}}{\sqrt{A(m+1) n_{\mathrm{eff}}}}\right), \\
& P(M \geqslant m)=P\left(n^{(m)} \geqslant n_{\mathrm{eff}}\right)=Q\left(\frac{m n_{\mathrm{eff}}-n}{\sqrt{A(m) n_{\mathrm{eff}}}}\right),
\end{aligned}
$$

where $Q(\cdot)$ is complementary cumulative distribution function (CCDF) of Gaussian distribution. Therefore,

$$
P\left(M \leqslant\left\lfloor M_{0}\right\rfloor-2\right)=Q\left(\frac{n-\left(\left\lfloor M_{0}\right\rfloor-1\right) n_{\mathrm{eff}}}{\sqrt{A\left(\left\lfloor M_{0}\right\rfloor-1\right) n_{\mathrm{eff}}}}\right) \leqslant Q\left(\sqrt{\frac{n_{\mathrm{eff}}}{A\left(M_{0}-1\right)}}\right)
$$


and

$$
P\left(M \geqslant\left\lfloor M_{0}\right\rfloor+2\right)=Q\left(\frac{\left(\left\lfloor M_{0}\right\rfloor+2\right) n_{\mathrm{eff}}-n}{\sqrt{A\left(\left\lfloor M_{0}\right\rfloor+2\right) n_{\mathrm{eff}}}}\right) \leqslant Q\left(\sqrt{\frac{n_{\mathrm{eff}}}{A\left(M_{0}+1\right)}}\right),
$$

where the floor function $\lfloor x\rfloor$ denotes the largest integer that is not greater than $x$. For $m<M_{0}+2, A(m)$ can be upper-bounded as

$$
A(m) \leqslant(m-1)-\int_{M_{0}+2-m}^{M_{0}} \frac{1}{x} \mathrm{~d} x=(m-1)-\ln \frac{M_{0}}{M_{0}+2-m} .
$$

Then, for $n$ and $n_{\text {eff }}$ in the range of our interests, we have

$$
P\left(M \geqslant\left\lfloor M_{0}\right\rfloor+2\right) \leqslant Q\left(\sqrt{\frac{n_{\mathrm{eff}}}{A\left(M_{0}+1\right)}}\right) \leqslant Q\left(\sqrt{\frac{n_{\mathrm{eff}}}{M_{0}-\ln M_{0}}}\right) \approx 0 .
$$

Similarly, we have $P\left(M \leqslant\left\lfloor M_{0}\right\rfloor-2\right) \approx 0$. Thus, the pmf of $M$ are mostly concentrated at 3 points: $\left\lfloor\frac{n}{n_{\text {eff }}}\right\rfloor-1,\left\lfloor\frac{n}{n_{\text {eff }}}\right\rfloor$, and $\left\lfloor\frac{n}{n_{\text {eff }}}\right\rfloor+1$. Monte Carlo simulation results also verify this conclusion. Therefore, we have the lower bound on $\mathrm{E}[M]$ as

$$
\mathrm{E}[M] \geqslant\left\lfloor\frac{n-n_{\mathrm{eff}}}{n_{\mathrm{eff}}}\right\rfloor
$$

Since $E_{0} /\left(r_{c}^{\beta-1}+\eta\right)$ is a decreasing function in $r_{c}$, using (24), (25) and (27), we obtain a new lower bound on $T_{\omega}^{\max }$ as

$$
\begin{aligned}
T_{\omega}^{L}= & \max _{n_{\mathrm{eff}}} T_{1}\left(n_{\mathrm{eff}}\right) \equiv \max _{n_{\mathrm{eff}}}\left\lfloor\frac{n-n_{\mathrm{eff}}}{n_{\mathrm{eff}}}\right\rfloor \cdot \frac{E_{0}}{\left(\frac{1}{\pi n_{\mathrm{eff}}} \ln \frac{n_{\mathrm{eff}}}{1-\omega-g\left(n_{\mathrm{eff}}\right)}\right)^{(\beta-1) / 2}+\eta} \\
& \text { subject to } n_{\mathrm{eff}}>g^{-1}(1-\omega)
\end{aligned}
$$

where $g^{-1}(\cdot)$ is the inverse function of $g\left(n_{\mathrm{eff}}\right)$. By temporarily removing the floor function $\lfloor\cdot\rfloor$, we have the following convex optimization problem (given $\beta>3$ ):

$$
\begin{aligned}
& \max _{n_{\mathrm{eff}}} \frac{E_{0}\left(n-n_{\mathrm{eff}}\right)}{n_{\mathrm{eff}}\left(\frac{1}{\pi n_{\mathrm{eff}}} \ln \frac{n_{\mathrm{eff}}}{1-\omega-g\left(n_{\mathrm{eff}}\right)}\right)^{(\beta-1) / 2}+\eta \cdot n_{\mathrm{eff}}}, \\
& \text { subject to } \quad n_{\mathrm{eff}}>g^{-1}(1-\omega) .
\end{aligned}
$$

The verification of the concavity of the objective function is omitted due to space limitation.

The convex optimization problem defined in (28) can be solved easily by numerical methods. Suppose that the solution of such problem is $\bar{n}_{\text {eff }}$, then

$$
T_{\omega}^{L}=\max \left\{T_{1}\left(n_{\mathrm{eff}}^{1}\right), T_{1}\left(n_{\mathrm{eff}}^{2}\right)\right\}
$$


where $n_{\text {eff }}^{1}=n /\left\lfloor\frac{n}{\left.\bar{n}_{\text {eff }}\right\rfloor}\right\rfloor, n_{\text {eff }}^{2}=n /\left\lceil\frac{n}{\bar{n}_{\text {eff }}}\right\rceil$, and $\lceil x\rceil$ denotes the smallest integer that is equal to or greater than $x$. We can also obtain the corresponding $n_{\mathrm{eff}}^{L}$ and $r_{c}^{L}$ as

$$
n_{\mathrm{eff}}^{L}=\underset{n_{\mathrm{eff}}^{1}, n_{\mathrm{eff}}^{2}}{\arg \max } T_{1}\left(n_{\mathrm{eff}}\right), \quad r_{c}^{L}=\sqrt{\left[\ln \left(n_{\mathrm{eff}}^{L} /\left(1-\omega-g\left(n_{\mathrm{eff}}^{L}\right)\right)\right)\right] /\left(\pi n_{\mathrm{eff}}^{L}\right)} .
$$

Upper bound. Next, we present an approximate upper bound on $T_{\omega}^{\max }$. Relaxing the constraint in (20) with the upper bound in (21), we obtain the relaxed constraints as

$$
P(A) \geqslant \omega, P(B) \geqslant \omega .
$$

Then, we use the lower bounds to approximate $P(A)$ and $P(B)$ as

$$
P(A) \approx P_{l} \approx 1-g\left(n_{\mathrm{eff}}^{(i)}\right), P(B) \approx 1-n_{\mathrm{eff}}^{(i)} e^{-n_{\mathrm{eff}}^{(i)} \pi\left(r_{c}^{(i)}\right)^{2}} .
$$

Next, we assume that the number of active sensors in round $i$ is approximately equal to $n_{\mathrm{eff}}^{(i)}$. Then the maximum number of rounds, $M$, is a deterministic quantity, and satisfies the constraint $\sum_{i=1}^{M} n_{\text {eff }}^{(i)} \leqslant n$. Using (30) and (31), we obtain an approximate upper bound on $T_{\omega}^{\max }$ by solving the following optimization problem:

$$
\begin{aligned}
& \max _{n_{\text {eff }}^{(i)}} \sum_{i=1}^{M} \frac{E_{0}}{\left(\frac{1}{\pi n_{\text {eff }}^{(i)}} \ln \frac{n_{\text {eff }}^{(i)}}{\omega}\right)^{(\beta-1) / 2}+\eta}, \\
& \text { subject to } \quad n_{\text {eff }}^{(i)} \geqslant g^{-1}(1-\omega), \sum_{i=1}^{M} n_{\text {eff }}^{(i)} \leqslant n .
\end{aligned}
$$

It is easy to verify that, given $M$, (32) is a convex optimization problem. By Lagrange multiplier, we obtain a new upper bound on $T_{\omega}^{\max }$ as

$$
\begin{aligned}
& T_{\omega}^{U}=\max _{n_{\text {eff }}} T_{2}\left(n_{\text {eff }}\right) \equiv \max _{n_{\text {eff }}}\left\lfloor\frac{n}{\left.n_{\text {eff }}\right\rfloor \cdot \frac{E_{0}}{\left(\frac{1}{\pi n_{\text {eff }}} \ln \frac{n_{\text {eff }}}{1-\omega}\right)^{(\beta-1) / 2}+\eta},}\right. \\
& \text { subject to } n_{\text {eff }}^{(i)} \geqslant g^{-1}(1-\omega) .
\end{aligned}
$$

Similarly, we temporarily remove the floor function $\lfloor\cdot\rfloor$. It is easy to verify that the resulting optimization problem is a convex problem. Suppose that the solution of such problem is $\tilde{n}_{\text {eff }}$, then

$$
\begin{aligned}
& T_{\omega}^{U}=\max \left\{T_{2}\left(n_{\text {eff }}^{1}\right), T_{2}\left(n_{\text {eff }}^{2}\right)\right\}, \quad n_{\text {eff }}^{U}=\underset{n_{\text {eff }}^{1}, n_{\text {eff }}^{2}}{\arg \max } T_{2}\left(n_{\text {eff }}\right), \quad \text { and } \\
& r_{c}^{U}=\sqrt{\left[\ln \left(n_{\text {eff }}^{U} /(1-\omega)\right)\right] /\left(\pi n_{\text {eff }}^{U}\right)},
\end{aligned}
$$

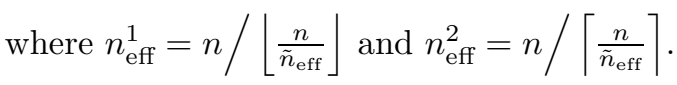


As an example, we let $E_{0}=1, \beta=3.5, \eta=0.001, \omega=0.92$, and $k=1$. Numerical results show that the relative difference between the lower bound $\left(T_{\omega}^{L}\right)$ and the upper bound $\left(T_{\omega}^{U}\right)$ is at the level of $10 \%$ for $n$ from 10000 to 40000 , which suggests that the derived lower bound is a good approximation of the optimal $\omega$-lifetime of the sensor network.

\subsection{PIS Scheme Design}

We propose to choose the operational parameters for the PIS scheme according to the derived lower bound on the optimal $\omega$-lifetime, i.e., choosing $p^{(i)}$ and $r_{c}^{(i)}$ for round $i$ as

$$
p^{(i)}=\min \left\{n_{\mathrm{eff}}^{L} / n^{(i)}, 1\right\}, r_{c}^{(i)}=r_{c}^{L},
$$

where $n^{(i)}$ is the number of alive sensors at the beginning of round $i(i \geqslant 1)$, and $n_{\text {eff }}^{L}$ and $r_{c}^{L}$ are given in (29). Obviously, (34) provides a centralized solution, since $n^{(i)}$ is a global information. At the beginning of each round, such information is required for each alive sensor to calculate $p^{(i)}$ online.

In resource-constrained wireless sensor networks, we always prefer distributed solutions. In our case, distributed solutions mean that the choices of $p^{(i)}$ 's should be independent of $n^{(i)}$. As shown in Section 4.1, the expected number of active sensors in each round, $n^{(i)} p^{(i)}$, is the key parameter to determine whether the network satisfies the " $P_{\mathrm{c} \& c} \geqslant \omega$ " requirement or not. According to the lower bound on the optimal $\omega$-lifetime, we define outage of round $i$ as the event that $n^{(i)} p^{(i)}<n_{\text {eff }}^{L}$, which means that the " $P_{\text {c\&c }} \geqslant \omega$ " requirement can not be satisfied at round $i$. The probability that an outage occurs at round $i$ is denoted by $P_{\text {out }}^{(i)}$. For the centralized solution in (34), $P_{\text {out }}^{(i)}$ is always 0 for the rounds that $n^{(i)} \geqslant n_{\text {eff. }}^{L}$

As an approximation to (34), we propose a distributed solution as follows:

$$
p^{(i)}=\left\{\begin{array}{ll}
\frac{n_{\text {eff }}^{L}\left(1+\epsilon^{(i)}\right)}{n-n_{\text {eff }}^{L} \sum_{l=1}^{i-1}\left(1+\epsilon^{(l)}\right)} & 1 \leqslant i<M \\
1 & i=M
\end{array}, \quad r_{c}^{(i)}=r_{c}^{L},\right.
$$

where $M$ is the maximum number of rounds, $\epsilon^{(1)}=0$, and for $1<i<M, \epsilon^{(i)}$ 's are chosen such that

$$
P_{\text {out }}^{(i)}=P\left(n^{(i)} p^{(i)}<n_{\text {eff }}^{L}\right)=\delta,
$$

where $\delta>0$ is a pre-defined small quantity.

With the choice of $p^{(i)}$ in (35), where $1<i<M$, we can approximate $n^{(i)}$ as a Gaussian random variable by the central limit theorem:

$$
n^{(i)} \sim \mathcal{N}\left(n-n_{\text {eff }}^{L} \sum_{l=1}^{i-1}\left(1+\epsilon^{(l)}\right), n_{\text {eff }}^{L} \sum_{l=1}^{i-1}\left(1+\epsilon^{(l)}\right)\left(1-p^{(l)}\right)\right) .
$$


Then, $\epsilon^{(i)}$ 's in (35) can be calculated recursively according to

$$
\epsilon^{(i)}=\left\{\begin{array}{ll}
0 & i=1 \\
\frac{Q^{-1}(\delta)}{a^{(i)}-Q^{-1}(\delta)} & 1<i<M
\end{array},\right.
$$

and

$$
a^{(i)}=\frac{n-n_{\mathrm{eff}}^{L} \sum_{l=1}^{i-1}\left(1+\epsilon^{(l)}\right)}{\sqrt{n_{\mathrm{eff}}^{L} \sum_{l=1}^{i-1}\left(1+\epsilon^{(l)}\right)\left(1-p^{(l)}\right)}},
$$

where $Q^{-1}(\cdot)$ is the inverse function of $Q(\cdot)$. The maximum number of rounds $(M)$ is defined as

$$
M=\underset{i>1}{\arg \max }\left\{a^{(i)}>0\right\} .
$$

The idea of this distributed solution is to use the expected number of alive sensors to replace $n^{(i)}$ in (34), and increase the expected number of active sensors slightly by $n_{\text {eff }}^{L} \epsilon^{(i)}$ such that the outage probability $\left(P_{\text {out }}^{(i)}\right)$ can be controlled at a given level $(\delta)$. In fact, this algorithm sacrifices the total number of rounds, equivalently network's lifetime, to achieve the distributed property.

\section{Simulation Results}

In this section, we use simulation results to demonstrate the performance of the proposed PIS scheduling schemes. The performance criterion is the $\omega$-lifetime of the network. As a comparison, we include the results of a PIS-like scheme that simply fixes the communication range to be twice the mean of the sensing radius $\left(r_{c}=2 r_{0}\right)$, and $n_{\text {eff }}^{(i)}$ to be $n_{\text {eff }}^{A}$, regardless of $i$. Here, $n_{\text {eff }}^{A}$ is obtained by solving the following equation: $P(A)=\omega$, where $P(A)$ is given in (31). We call this scheme the PIS-naive scheme.

We simulate a square sensing field $\mathcal{D}$ of unit area in which $n$ sensors are deployed independently and uniformly. The sensing radius $r_{s}$ is assumed to be a uniformly distributed random variable on $[0.0384,0.1216]$, which corresponds to $r_{0}=0.08$ and $\sigma_{s}=0.3$. Let $E_{0}=1, \beta=3.5, \eta=0.001, \omega=0.92$, and $k=1$, i.e, we considerer 1-coverage as an example. With this network setup, the centralized and distributed PIS schemes select $p^{(i)}$ and $r_{c}^{(i)}$ according to (34) and (35), respectively. For the distributed PIS scheme, the outage probability threshold $(\delta)$ is set to $10^{-2}$. The PIS-naive scheme selects $p^{(i)}$ according to (34) with $n_{\text {eff }}^{L}$ replaced by $n_{\text {eff }}^{A}$.

First, we simulate the operation of a network with $n=10000$ using different scheduling schemes. We divide the field $\mathcal{D}$ into a grid of size $62 \times 62$, and approximate that the field $\mathcal{D}$ is $k$-covered if all grid points are $k$-covered. For the connectivity, we approximate that the network is connected if there is no isolated active sensors. The torus convention is also employed for simulations to avoid 


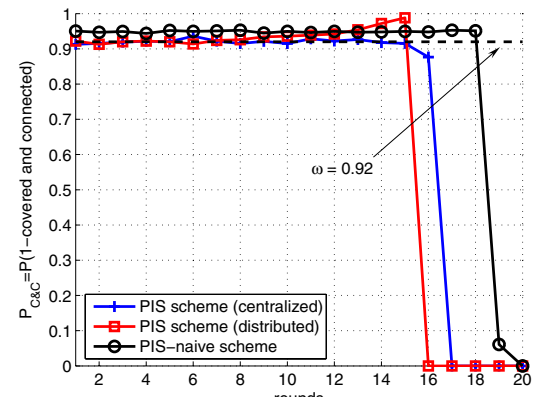

Fig. 1. Three snapshots of the network operation

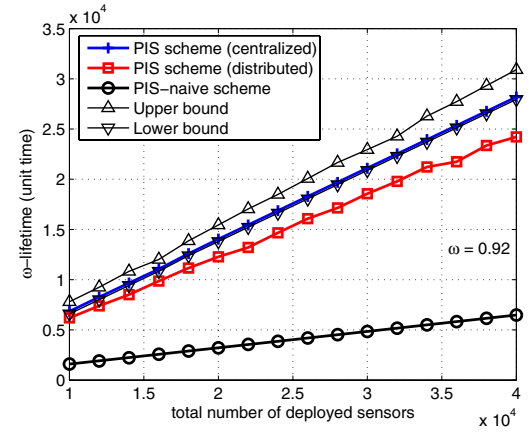

Fig. 2. $\omega$-lifetime comparison for different scheduling schemes

edge effects. Then, $P_{c \& c}$ at each round of the network operation is estimated as follows: given a deployment, the network is operated according to the particular scheduling scheme until the batteries of all sensors die out. Repeat this experiment 2500 times with the same deployment. For round $i$ of experiment $j$, define $\delta_{j}^{i}=1$ if the field $\mathcal{D}$ is $k$-covered and active sensors are connected, 0 otherwise. Then, $P_{\mathrm{c} \& \mathrm{c}}$ of round $i$ can be estimated as $P_{\mathrm{c} \& \mathrm{c}}^{i}=\frac{1}{2500} \sum_{j=1}^{2500} \delta_{j}^{i}$.

Fig. 1 shows three snapshots of the network operation using PIS-naive scheme, centralized and distributed PIS scheduling schemes, respectively. It is seen that all scheduling schemes can guarantee that the network satisfies the connectivity and $k$-coverage requirements as long as the expected number of active sensors is no less than $n_{\text {eff }}^{L}$. Therefore, in the simulation of the network's $\omega$-lifetime, we only need to simulate how many rounds a network can operate properly following a particular scheduling scheme. Notice that the PIS-naive scheme can operate more rounds than the PIS schemes. However, each round is shorter in the PISnaive scheme, since $r_{c}$ is not optimally selected. As seen in the next simulation, the PIS schemes have longer $\omega$-lifetime than the PIS-naive scheme.

Second, we compare the $\omega$-lifetime of a network using different scheduling schemes with $n$ from 10000 to 40000, and the results are plotted in Fig. 2, The derived lower bound and upper bound for the PIS scheme are also shown in the figure. The estimate of the $\omega$-lifetime is calculated as:

$$
\widehat{T}_{\text {net }}=\frac{1}{N} \sum_{j=1}^{N} M_{j} \cdot T_{0}\left(r_{c}\right),
$$

where $N$ is the number of Monte Carlo realizations (we set $N$ to 1000 in this simulation), $T_{0}\left(r_{c}\right)$ is the duration of each round defined in (17), and $M_{j}$ is the number of rounds the network can operate properly at the $j$-th Monte Carlo realization. At each Monte Carlo realization, the network is said to operate properly at round $i$ if the expected number of active sensors at round $i$ is at least $n_{\mathrm{eff}}^{L}$, i.e.,

$$
n^{(i)} p^{(i)} \geqslant n_{\mathrm{eff}}^{L}
$$


We observe that for the centralized PIS scheme, the simulation result is very close to the theoretical lower bound, $T_{\omega}^{L}$, which was derived in Section 4 By comparing the PIS schemes and the PIS-naive scheme, we clearly see that the $\omega$-lifetime's of both centralized and distributed PIS schemes are much longer than that of the PIS-naive scheme, and the differences become larger with more deployed sensors. Such fact demonstrates the importance of joint optimization of lifetime, connectivity, and coverage. We also see that the $\omega$-lifetime of the distributed PIS scheme is close to that of the centralized one, which suggests that the distributed PIS scheme is a good choice for real applications.

\section{Conclusions and Future Work}

In this paper, we investigate the fundamental limits of a wireless sensor network's lifetime under connectivity and $k$-coverage constraints. The contributions of the paper are twofold. First, we study the lifetime of a wireless sensor network from a novel probabilistic perspective and introduce a new concept, called network's $\omega$-lifetime, which is defined as the expectation of the time interval during which the probability of guaranteeing connectivity and $k$-coverage simultaneously is at least $\omega$. Second, we propose PIS (Pre-planned Independent Sleeping) as a near-optimal scheduling scheme to maximize the $\omega$-lifetime of a finite-size wireless sensor network, describe a possible distributed implementation of the PIS scheme, and demonstrate the PIS performance by simulation results.

Future work includes extending the analysis to more generic and realistic scenarios such as when only a portion of the sensing field needs to be $k$-covered, or when the sensing field is of irregular shape, or when the communication radius is also a random variable.

\section{References}

1. I. Akyildiz, W. Su, Y. Sankarasubramaniam, and E. Cayirci, "A survey on sensor networks," IEEE Communication Magazine, vol. 40, no. 8, pp. 102-114, Aug. 2002.

2. D. Estrin, R. Govindan, J. Heidemann, and S. Kumar, "Next century challenges: scalable coordination in sensor networks," in Proc. ACM MobiCom'99, Seattle, WA, 1999, pp. 263-270.

3. P. Gupta and P. Kumar, "Critical power for asymptotic connectivity," in Proc. the 37th IEEE Conference on Decision and Control, vol. 1, 1998, pp. 1106-1110.

4. D. Miorandi and E. Altman, "Coverage and connectivity of ad hoc networks in presence of channel randomness," in Proc. IEEE INFOCOM'05, Miami, FL, Mar. 2005, pp. 491-502.

5. X. Wang, G. Xing, Y. Zhang, C. Lu, R. Pless, and C. Gill, "Integrated coverage and connectivity configuration in wireless sensor networks," in Proc. ACM SenSys'03, Los Angeles, CA, 2003, pp. 28-39.

6. B. Liu and D. Towsley, "A study of the coverage of large-scale sensor networks," in Proc. IEEE MASS'04, Fort Lauderdale, FL, Oct. 2004, pp. 475-483.

7. S. Shakkottai, R. Srikant, and N. Shroff, "Unreliable sensor grids: coverage, connectivity and diameter," in Proc. IEEE INFOCOM'03, vol. 2, 2003, pp. 1073-1083. 
8. S. Kumar, T. Lai, and J. Balogh, "On k-coverage in a mostly sleeping sensor network," in Proc. ACM MobiCom'04, Philadelphia, PA, 2004, pp. 144-158.

9. F. Ye, G. Zhong, J. Cheng, S. Lu, and L. Zhang, "PEAS: a robust energy conserving protocol for long-lived sensor networks," in Proc. IEEE ICDCS'03, Providence, RI, May 2003, pp. 28-37.

10. H. Zhang and J. Hou, "On deriving the upper bound of $\alpha$-lifetime for large sensor networks," in Proc. ACM MobiHoc'04, Roppongi Hills, Tokyo, Japan, 2004, pp. $121-132$.

11. Y. Xu, J. Heidemann, and D. Estrin, "Geography-informed energy conservation for ad hoc routing," in Proc. ACM MobiCom'01, Rome, Italy, July 2001.

12. C. Hsin and M. Liu, "Network coverage using low duty-cycled sensors: random and coordinated sleep algorithms," in Proc. IEEE IPSN'04, Berkeley, CA, Apr. 2004, pp. 433-442.

13. C. Gui and P. Mohapatra, "Power convervation and quality of surveillance in target tracking sensor networks," in Proc. ACM MobiCom'04, Philadelphia, PA, Sept. 2004.

14. P. Hall, Introduction to the Theory of Coverage Process. John Wiley and Sons, 1988.

15. W. Mo, D. Qiao, and Z. Wang, "Mostly sleeping wireless sensor networks: connectivity, $k$-coverage, and lifetime," Electrical and Computer Engineering Department, Iowa State University, Tech. Rep., 2005.

16. P. Billingsley, Probability and Measure. New York: Wiley, 1979.

17. T. Rappaport, Wireless Communications: Principles and Practice. Prentice Hall, 2001.

18. D. Blough and P. Santi, "Investigating upper bounds on network lifetime extension for cell-based energy conservation techniques in stationary ad hoc networks," in Proc. MobiCom'02, 2002, pp. 183-192. 